\title{
SOME NEW ČEBYŠEV TYPE INEQUALITIES
}

\author{
Fiza Zafar, NAZir Ahmad Mir, AND Arif RAFiQ
}

\begin{abstract}
Some new Čebyšev type inequalities have been developed by working on functions whose first derivatives are absolutely continuous and the second derivatives belong to the usual Lebesgue space $L_{\infty}[a, b]$. A unified treatment of the special cases is also given.
\end{abstract}

\section{Introduction}

For two measurable functions $f, g:[a, b] \rightarrow \mathbb{R}$, we define the functional, (1.1)

$$
T(f, g ; a, b):=\frac{1}{b-a} \int_{a}^{b} f(x) g(x) d x-\left(\frac{1}{b-a} \int_{a}^{b} f(x) d x\right)\left(\frac{1}{b-a} \int_{a}^{b} g(x) d x\right),
$$

which in literature is called the Čebyšev functional, provided the integrals in (1.1) exist.

Moreover, in 1882, P. L. Čebyšev (see [4], p. 297) proved that, if $f^{\prime}, g^{\prime} \in$ $L_{\infty}[a, b]$, then

$$
|T(f, g ; a, b)| \leq \frac{1}{12}(b-a)^{2}\left\|f^{\prime}\right\|_{\infty}\left\|g^{\prime}\right\|_{\infty} .
$$

In the recent past, Čebyšev functional has remained an area of special interest for many researchers and has yielded many variants and generalizations in the field of inequalities. It has also played a key role in obtaining some new inequalities of Ostrowski type, for example, Ostrowski-Grüss type, OstrowskiČebyšev type, etc. The research papers $[1,2,5]$ cover a comprehensive literature on the generalizations of Čebyšev functional and its associated bounds.

In [6], B. G. Pachpatte presented the following Čebyšev type inequality by using trapezoid like rules:

Theorem 1. Let $f, g:[a, b] \rightarrow \mathbb{R}$ be differentiable functions so that $f^{\prime}, g^{\prime}$ are absolutely continuous on $[a, b]$. Then

$$
|P(\bar{F}, \bar{G}, f, g)| \leq \frac{(b-a)^{4}}{144}\left\|f^{\prime \prime}-\left[f^{\prime} ; a, b\right]\right\|_{\infty}\left\|g^{\prime \prime}-\left[g^{\prime} ; a, b\right]\right\|_{\infty},
$$

Received February 13, 2008; Revised March 30, 2009.

2000 Mathematics Subject Classification. Primary 26D15; Secondary 26D20.

Key words and phrases. Čebyšev type inequalities, midpoint rule, trapezoid like rule. 
where

$$
\begin{aligned}
& \bar{F}=\frac{f(a)+f(b)}{2}-\frac{(b-a)^{2}}{12}\left[f^{\prime} ; a, b\right], \\
& \bar{G}=\frac{g(a)+g(b)}{2}-\frac{(b-a)^{2}}{12}\left[g^{\prime} ; a, b\right]
\end{aligned}
$$

and

$$
\begin{aligned}
P(\alpha, \beta, f, g)= & \alpha \beta-\frac{1}{b-a}\left(\alpha \int_{a}^{b} g(t) d t+\beta \int_{a}^{b} f(t) d t\right) \\
& +\left(\frac{1}{b-a} \int_{a}^{b} f(t) d t\right)\left(\frac{1}{b-a} \int_{a}^{b} g(t) d t\right), \\
{\left[f^{\prime} ; a, b\right]=} & \frac{f^{\prime}(b)-f^{\prime}(a)}{b-a} .
\end{aligned}
$$

Recently, in [3], Z. Liu presented the following generalization of (1.3):

Theorem 2. Let the assumptions of Theorem 1 hold. Then for any $\theta \in[0,1]$,

$$
\left|P\left(\bar{\Gamma}_{\theta}, \bar{\Delta}_{\theta}, f, g\right)\right| \leq(b-a)^{4} I^{2}(\theta)\left\|f^{\prime \prime}-\left[f^{\prime} ; a, b\right]\right\|_{\infty}\left\|g^{\prime \prime}-\left[g^{\prime} ; a, b\right]\right\|_{\infty},
$$

where

$$
I(\theta)=\left\{\begin{array}{c}
\frac{\theta^{3}}{3}-\frac{\theta}{8}+\frac{1}{24}, 0 \leq \theta \leq \frac{1}{2}, \\
\frac{1}{8}\left(\theta-\frac{1}{3}\right), \quad \frac{1}{2}<\theta \leq 1
\end{array}\right.
$$

and

$$
\begin{aligned}
\Gamma_{\theta} & =\frac{\theta}{2}[f(a)+f(b)]+(1-\theta) f\left(\frac{a+b}{2}\right), \\
\Delta_{\theta} & =\frac{\theta}{2}[g(a)+g(b)]+(1-\theta) g\left(\frac{a+b}{2}\right), \\
\bar{\Gamma}_{\theta} & =\Gamma_{\theta}+\frac{1}{24}(1-3 \theta)(b-a)^{2}\left[f^{\prime} ; a, b\right], \\
\bar{\Delta}_{\theta} & =\Delta_{\theta}+\frac{1}{24}(1-3 \theta)(b-a)^{2}\left[g^{\prime} ; a, b\right] .
\end{aligned}
$$

In this paper, we, by following an approach similar to that of [3] and [6], present some new Čebyšev type inequalities.

\section{Main results}

For suitable functions $f, g:[a, b] \rightarrow \mathbb{R}$ and $h \in[0,1]$, we use the following notations:

$$
\begin{aligned}
\bar{T}_{h, x}= & \frac{1}{2}(2-h) f(x)-(1-h)\left(x-\frac{a+b}{2}\right) f^{\prime}(x) \\
& +\frac{h}{2}\left(\frac{(x-a) f(a)+(b-x) f(b)}{b-a}\right),
\end{aligned}
$$




$$
\begin{aligned}
\bar{S}_{h, x}= & \frac{1}{2}(2-h) g(x)-(1-h)\left(x-\frac{a+b}{2}\right) g^{\prime}(x) \\
& +\frac{h}{2}\left(\frac{(x-a) g(a)+(b-x) g(b)}{b-a}\right), \\
\bar{H}_{h, x}= & (1-h) f(x)+h\left(\frac{(x-a) f(a)+(b-x) f(b)}{b-a}\right) \\
& -(1-h)^{2}\left(x-\frac{a+b}{2}\right) f^{\prime}(x) \\
& -\frac{h^{2}}{2}\left(\frac{(b-x)^{2} f^{\prime}(b)-(x-a)^{2} f^{\prime}(a)}{b-a}\right), \\
\bar{L}_{h, x}= & (1-h) g(x)+h\left(\frac{(x-a) g(a)+(b-x) g(b)}{b-a}\right) \\
& -(1-h)^{2}\left(x-\frac{a+b}{2}\right) g^{\prime}(x) \\
& -\frac{h^{2}}{2}\left(\frac{(b-x)^{2} g^{\prime}(b)-(x-a)^{2} g^{\prime}(a)}{b-a}\right), \\
T_{h, x}= & \bar{T}_{h, x}+\frac{1}{4}(2-3 h)\left[f^{\prime} ; a, b\right](b-a)^{2} \Delta(x), \\
S_{h, x}= & \bar{S}_{h, x}+\frac{1}{4}(2-3 h)\left[g^{\prime} ; a, b\right](b-a)^{2} \Delta(x), \\
H_{h, x}= & \bar{H}_{h, x}+\frac{1}{2}\left(3 h^{2}-3 h+1\right)\left[f^{\prime} ; a, b\right](b-a)^{2} \Delta(x),
\end{aligned}
$$

and

where

$$
L_{h, x}=\bar{L}_{h, x}+\frac{1}{2}\left(3 h^{2}-3 h+1\right)\left[g^{\prime} ; a, b\right](b-a)^{2} \Delta(x),
$$

$$
\Delta(x)=\frac{1}{12}+\left(\frac{x-\frac{a+b}{2}}{b-a}\right)^{2},
$$

and $\left[f^{\prime} ; a, b\right]$ is defined as above.

Theorem 3. Let the assumptions of Theorem 1 hold. Then for any $h \in[0,1]$,

$$
\begin{aligned}
& \left|P\left(T_{h, x}, S_{h, x}, f, g\right)\right| \\
\leq & \frac{1}{16} \omega^{2}(h)(b-a)^{4} \Delta^{2}(x)\left\|f^{\prime \prime}-\left[f^{\prime} ; a, b\right]\right\|_{\infty}\left\|g^{\prime \prime}-\left[g^{\prime} ; a, b\right]\right\|_{\infty},
\end{aligned}
$$

where $\Delta(x), T_{h, x}$ and $S_{h, x}$ are defined as above and

$$
\omega(h)=2 h^{3}-3 h+2 .
$$


Proof. We define the following kernel:

$$
K(x, t ; h)=\left\{\begin{array}{l}
\frac{1}{2}(t-a)(t-(1-h) a-h x), t \in[a, x], \\
\frac{1}{2}(t-b)(t-h x-(1-h) b), t \in(x, b] .
\end{array}\right.
$$

Through simple calculations, it can be shown that

$$
\begin{aligned}
& \frac{1}{b-a} \int_{a}^{b} f(t) d t-T_{h, x}=I\left(f^{\prime}, f^{\prime \prime} ; a, b\right), \\
& \frac{1}{b-a} \int_{a}^{b} g(t) d t-S_{h, x}=I\left(g^{\prime}, g^{\prime \prime} ; a, b\right),
\end{aligned}
$$

where

$$
I\left(f^{\prime}, f^{\prime \prime} ; a, b\right)=\frac{1}{b-a} \int_{a}^{b} K(x, t ; h)\left\{f^{\prime \prime}(t)-\left[f^{\prime} ; a, b\right]\right\} d t .
$$

Multiplying the left and right hand side of (2.4) and (2.5), we get,

$$
P\left(T_{h, x}, S_{h, x}, f, g\right)=I\left(f^{\prime}, f^{\prime \prime} ; a, b\right) I\left(g^{\prime}, g^{\prime \prime} ; a, b\right),
$$

implies

$$
\left|P\left(T_{h, x}, S_{h, x}, f, g\right)\right|=\left|I\left(f^{\prime}, f^{\prime \prime} ; a, b\right)\right|\left|I\left(g^{\prime}, g^{\prime \prime} ; a, b\right)\right| .
$$

Following an approach similar to [6], we have

$$
\begin{aligned}
\left|I\left(f^{\prime}, f^{\prime \prime} ; a, b\right)\right| & \leq \frac{1}{b-a} \int_{a}^{b}|K(x, t ; h)|\left|f^{\prime \prime}(t)-\left[f^{\prime} ; a, b\right]\right| d t \\
& \leq \frac{1}{b-a}\left\|f^{\prime \prime}(t)-\left[f^{\prime} ; a, b\right]\right\|_{\infty} \int_{a}^{b}|K(x, t ; h)| d t .
\end{aligned}
$$

Similarly, we have

$$
\left|I\left(g^{\prime}, g^{\prime \prime} ; a, b\right)\right| \leq \frac{1}{b-a}\left\|g^{\prime \prime}(t)-\left[g^{\prime} ; a, b\right]\right\|_{\infty} \int_{a}^{b}|K(x, t ; h)| d t .
$$

From the definition of $K(x, t ; h)$, it follows that

$$
\frac{1}{b-a} \int_{a}^{b}|K(x, t ; h)| d t=\frac{1}{4} \omega(h)(b-a)^{2} \Delta(x),
$$

where $\Delta(x)$ and $\omega(h)$ are defined by $(2.1)$ and $(2.3)$.

By using (2.6)-(2.9), (2.2) follows.

The following corollary of Theorem 3 holds:

Corollary 4. Let the assumptions of Theorem 1 hold. Then for any $h \in[0,1]$,

$$
\begin{aligned}
& \left|P\left(T_{h, \frac{a+b}{2}}, S_{h, \frac{a+b}{2}}, f, g\right)\right| \\
\leq & \frac{1}{2304} \omega^{2}(h)(b-a)^{4}\left\|f^{\prime \prime}-\left[f^{\prime} ; a, b\right]\right\|_{\infty}\left\|g^{\prime \prime}-\left[g^{\prime} ; a, b\right]\right\|_{\infty},
\end{aligned}
$$


where

$$
\begin{aligned}
T_{h, \frac{a+b}{2}}= & \frac{1}{2}(2-h) f\left(\frac{a+b}{2}\right)+\frac{h}{4}(f(a)+f(b)) \\
& +\frac{1}{48}(2-3 h)\left[f^{\prime} ; a, b\right](b-a)^{2}, \\
S_{h, \frac{a+b}{2}}= & \frac{1}{2}(2-h) g\left(\frac{a+b}{2}\right)+\frac{h}{4}(g(a)+g(b)) \\
& +\frac{1}{48}(2-3 h)\left[g^{\prime} ; a, b\right](b-a)^{2}
\end{aligned}
$$

and $\omega(h)$ is defined by $(2.3)$.

Remark 1 . It may observed that for $x=\frac{a+b}{2}$, the kernel defined in Theorem 3 takes the following form:

$$
K\left(\frac{a+b}{2}, t ; h\right)=\left\{\begin{array}{cl}
\frac{1}{2}(t-a)\left(t-\left(a+h \frac{b-a}{2}\right)\right), & t \in\left[a, \frac{a+b}{2}\right], \\
\frac{1}{2}(t-b)\left(t-\left(b-h \frac{b-a}{2}\right)\right), & t \in\left(\frac{a+b}{2}, b\right] .
\end{array}\right.
$$

The following special cases of Corollary 4 hold:

Remark 2. 1. For $h=0,(2.10)$ takes the form,

$$
\begin{aligned}
& \left|P\left(T_{0, \frac{a+b}{2}}, S_{0, \frac{a+b}{2}}, f, g\right)\right| \\
\leq & \frac{1}{576}(b-a)^{4}\left\|f^{\prime \prime}-\left[f^{\prime} ; a, b\right]\right\|\left\|_{\infty}\right\| g^{\prime \prime}-\left[g^{\prime} ; a, b\right] \|_{\infty},
\end{aligned}
$$

with

$$
T_{0, \frac{a+b}{2}}=f\left(\frac{a+b}{2}\right)+\frac{1}{24}\left[f^{\prime} ; a, b\right](b-a)^{2}
$$

and

$$
S_{0, \frac{a+b}{2}}=g\left(\frac{a+b}{2}\right)+\frac{1}{24}\left[g^{\prime} ; a, b\right](b-a)^{2} .
$$

2. For $h=1,(2.10)$ takes the form,

$$
\begin{aligned}
& \left|P\left(T_{1, \frac{a+b}{2}}, S_{1, \frac{a+b}{2}}, f, g\right)\right| \\
\leq & \frac{1}{2304}(b-a)^{4}\left\|f^{\prime \prime}-\left[f^{\prime} ; a, b\right]\right\|_{\infty}\left\|g^{\prime \prime}-\left[g^{\prime} ; a, b\right]\right\|_{\infty},
\end{aligned}
$$

where

$$
T_{1, \frac{a+b}{2}}=\frac{1}{4}\left(f(a)+2 f\left(\frac{a+b}{2}\right)+f(b)\right)-\frac{1}{48}\left[f^{\prime} ; a, b\right](b-a)^{2}
$$

and

$$
S_{1, \frac{a+b}{2}}=\frac{1}{4}\left(g(a)+2 g\left(\frac{a+b}{2}\right)+g(b)\right)-\frac{1}{48}\left[g^{\prime} ; a, b\right](b-a)^{2} .
$$


3. For $h=\frac{2}{3},(2.10)$ takes the form,

$$
\begin{aligned}
& \left|P\left(T_{\frac{2}{3}, \frac{a+b}{2}}, S_{\frac{2}{3}, \frac{a+b}{2}}, f, g\right)\right| \\
\leq & \frac{1}{6561}(b-a)^{4}\left\|f^{\prime \prime}-\left[f^{\prime} ; a, b\right]\right\|_{\infty}\left\|g^{\prime \prime}-\left[g^{\prime} ; a, b\right]\right\|_{\infty},
\end{aligned}
$$

where

$$
T_{\frac{2}{3}, \frac{a+b}{2}}=\frac{1}{6}\left(f(a)+4 f\left(\frac{a+b}{2}\right)+f(b)\right)
$$

and

$$
S_{\frac{2}{3}, \frac{a+b}{2}}=\frac{1}{6}\left(g(a)+4 g\left(\frac{a+b}{2}\right)+g(b)\right) .
$$

It may also be noted that $\omega(h)$ is minimum for $h=\frac{1}{\sqrt{2}}$.

Theorem 5. Let the assumptions of Theorem 1 hold. Then for any $h \in[0,1]$,

$$
\begin{aligned}
& \left|P\left(H_{h, x}, L_{h, x}, f, g\right)\right| \\
\leq & \frac{1}{4} \eta^{2}(h)(b-a)^{4} \Delta^{2}(x)\left\|f^{\prime \prime}-\left[f^{\prime} ; a, b\right]\right\|_{\infty}\left\|g^{\prime \prime}-\left[g^{\prime} ; a, b\right]\right\|_{\infty},
\end{aligned}
$$

where $\Delta(x), H_{h, x}$ and $L_{h, x}$ are defined as above and

$$
\eta(h)=3 h^{2}-3 h+1 .
$$

Proof. We define the following kernel

$$
K_{1}(x, t ; h)=\left\{\begin{array}{l}
\frac{1}{2}(t-(1-h) a-h x)^{2}, t \in[a, x], \\
\frac{1}{2}(t-h x-(1-h) b)^{2}, t \in(x, b] .
\end{array}\right.
$$

Through simple calculations, it can be shown that

$$
\begin{aligned}
& \frac{1}{b-a} \int_{a}^{b} f(t) d t-H_{h, x}=J\left(f^{\prime}, f^{\prime \prime} ; a, b\right), \\
& \frac{1}{b-a} \int_{a}^{b} g(t) d t-L_{h, x}=J\left(g^{\prime}, g^{\prime \prime} ; a, b\right),
\end{aligned}
$$

where

$$
J\left(f^{\prime}, f^{\prime \prime} ; a, b\right)=\frac{1}{b-a} \int_{a}^{b} K_{1}(x, t ; h)\left\{f^{\prime \prime}(t)-\left[f^{\prime} ; a, b\right]\right\} d t .
$$

Multiplying the left and right hand side of (2.16) and (2.17), we get,

$$
P\left(H_{h, x}, L_{h, x}, f, g\right)=J\left(f^{\prime}, f^{\prime \prime} ; a, b\right) J\left(g^{\prime}, g^{\prime \prime} ; a, b\right),
$$

implies

$$
\left|P\left(H_{h, x}, L_{h, x}, f, g\right)\right|=\left|J\left(f^{\prime}, f^{\prime \prime} ; a, b\right)\right|\left|J\left(g^{\prime}, g^{\prime \prime} ; a, b\right)\right| .
$$

Following an approach similar to [6], we calculate

$$
\left|J\left(f^{\prime}, f^{\prime \prime} ; a, b\right)\right| \leq \frac{1}{b-a} \int_{a}^{b}\left|K_{1}(x, t ; h)\right|\left|f^{\prime \prime}(t)-\left[f^{\prime} ; a, b\right]\right| d t
$$




$$
\leq \frac{1}{b-a}\left\|f^{\prime \prime}(t)-\left[f^{\prime} ; a, b\right]\right\|_{\infty} \int_{a}^{b}\left|K_{1}(x, t ; h)\right| d t .
$$

Similarly, we have

$$
\left|J\left(g^{\prime}, g^{\prime \prime} ; a, b\right)\right| \leq \frac{1}{b-a}\left\|g^{\prime \prime}(t)-\left[g^{\prime} ; a, b\right]\right\|_{\infty} \int_{a}^{b}\left|K_{1}(x, t ; h)\right| d t .
$$

From the definition of $K_{1}(x, t ; h)$, it follows that

$$
\frac{1}{b-a} \int_{a}^{b}\left|K_{1}(x, t ; h)\right| d t=\frac{1}{2} \eta(h)(b-a)^{2} \Delta(x),
$$

where $\Delta(x)$ and $\eta(h)$ are defined by (2.1) and (2.15).

Therefore (2.14) follows directly from (2.18)-(2.21).

The following corollary of Theorem 5 holds:

Corollary 6. Let the assumptions of Theorem 1 hold. Then for any $h \in[0,1]$,

$$
\begin{aligned}
& \left|P\left(H_{h, \frac{a+b}{2}}, L_{h, \frac{a+b}{2}}, f, g\right)\right| \\
\leq & \frac{1}{576} \eta^{2}(h)(b-a)^{4}\left\|f^{\prime \prime}-\left[f^{\prime} ; a, b\right]\right\|_{\infty}\left\|g^{\prime \prime}-\left[g^{\prime} ; a, b\right]\right\|_{\infty},
\end{aligned}
$$

where

$$
H_{h, \frac{a+b}{2}}=(1-h) f\left(\frac{a+b}{2}\right)+\frac{h}{2}(f(a)+f(b))+\frac{1}{24}(1-3 h)(b-a)^{2}\left[f^{\prime} ; a, b\right]
$$

and

$$
L_{h, \frac{a+b}{2}}=(1-h) g\left(\frac{a+b}{2}\right)+\frac{h}{2}(g(a)+g(b))+\frac{1}{24}(1-3 h)(b-a)^{2}\left[g^{\prime} ; a, b\right],
$$

$\eta(h)$ is defined by $(2.15)$.

Remark 3. It may observed that for $x=\frac{a+b}{2}$, the kernel defined in Theorem 5 takes the following form:

$$
K_{1}\left(\frac{a+b}{2}, t ; h\right)=\left\{\begin{array}{c}
\frac{1}{2}\left(t-\left(a+h \frac{b-a}{2}\right)\right)^{2}, t \in\left[a, \frac{a+b}{2}\right], \\
\frac{1}{2}\left(t-\left(b-h \frac{b-a}{2}\right)\right)^{2}, t \in\left(\frac{a+b}{2}, b\right] .
\end{array}\right.
$$

The following special cases of Corollary 6 hold:

Remark 4. 1. For $h=0,(2.22)$ takes the form,

$$
\begin{aligned}
& \left|P\left(H_{0, \frac{a+b}{2}}, L_{0, \frac{a+b}{2}}, f, g\right)\right| \\
\leq & \frac{1}{576}(b-a)^{4}\left\|f^{\prime \prime}-\left[f^{\prime} ; a, b\right]\right\|_{\infty}\left\|g^{\prime \prime}-\left[g^{\prime} ; a, b\right]\right\|_{\infty},
\end{aligned}
$$

with

$$
H_{0, \frac{a+b}{2}}=f\left(\frac{a+b}{2}\right)+\frac{1}{24}\left[f^{\prime} ; a, b\right](b-a)^{2}
$$


and

$$
L_{0, \frac{a+b}{2}}=g\left(\frac{a+b}{2}\right)+\frac{1}{24}\left[g^{\prime} ; a, b\right](b-a)^{2} .
$$

2. For $h=1,(2.22)$ takes the form,

$$
\begin{aligned}
& \left|P\left(H_{1, \frac{a+b}{2}}, L_{1, \frac{a+b}{2}}, f, g\right)\right| \\
\leq & \frac{1}{576}(b-a)^{4}\left\|f^{\prime \prime}-\left[f^{\prime} ; a, b\right]\right\|_{\infty}\left\|g^{\prime \prime}-\left[g^{\prime} ; a, b\right]\right\|_{\infty},
\end{aligned}
$$

where

$$
H_{1, \frac{a+b}{2}}=\frac{1}{2}(f(a)+f(b))-\frac{1}{12}\left[f^{\prime} ; a, b\right](b-a)^{2}
$$

and

$$
L_{1, \frac{a+b}{2}}=\frac{1}{2}(g(a)+g(b))-\frac{1}{12}\left[g^{\prime} ; a, b\right](b-a)^{2} .
$$

3. For $h=\frac{1}{2},(2.22)$ takes the form,

$$
\begin{aligned}
& \left|P\left(H_{\frac{1}{2}, \frac{a+b}{2}}, L_{\frac{1}{2}, \frac{a+b}{2}}, f, g\right)\right| \\
\leq & \frac{1}{9216}(b-a)^{4}\left\|f^{\prime \prime}-\left[f^{\prime} ; a, b\right]\right\|_{\infty}\left\|g^{\prime \prime}-\left[g^{\prime} ; a, b\right]\right\|_{\infty},
\end{aligned}
$$

where

$$
H_{\frac{1}{2}, \frac{a+b}{2}}=\frac{1}{4}\left(f(a)+2 f\left(\frac{a+b}{2}\right)+f(b)\right)-\frac{1}{48}\left[f^{\prime} ; a, b\right](b-a)^{2}
$$

and

$$
L_{\frac{1}{2}, \frac{a+b}{2}}=\frac{1}{4}\left(g(a)+2 g\left(\frac{a+b}{2}\right)+g(b)\right)-\frac{1}{48}\left[g^{\prime} ; a, b\right](b-a)^{2} .
$$

It may also be noted that $\eta(h)$ is minimum for $h=\frac{1}{2}$.

\section{References}

[1] P. Cerone and S. S. Dragomir, Generalisations of the Grüss, Chebychev and Lupaş inequalities for integrals over different intervals, Int. J. Appl. Math. 6 (2001), no. 2, 117128.

[2] ㄴ. New bounds for the Čebyčev functional, Appl. Math. Lett. 18 (2005), no. 6, 603-611.

[3] Z. Liu, Generalizations of some new Čebyšev type inequalities, J. Inequal. Pure Appl. Math. 8 (2007), no. 1, Article 13, 6 pp.

[4] D. S. Mitrinović, J. E. Pečarić, and A. M. Fink Classical and New Inequalities in Analysis, Mathematics and its Applications (East European Series), 61. Kluwer Academic Publishers Group, Dordrecht, 1993.

[5] B. G. Pachpatte, On Ostrowski-Grüss-Čeby šev type inequalities for functions whose modulus of derivatives are convex, J. Inequal. Pure Appl. Math. 6 (2005), no. 4, Article 128, 15 pp.

[6] _ _ New Čebyšev type inequalities via trapezoidal-like rules, J. Inequal. Pure Appl. Math. 7 (2006), no. 1, Article 31, 6 pp. 
FIZA ZAFAR

Centre for Advanced Studies in Pure and Applied Mathematics

BAHAUDdIN ZAKARIYA UNIVERSITY

Multan 60800, Pakistan

E-mail address: fizazafar@gmail.com

NAZIR AHMAD Mir

Department of Mathematics

COMSATS Institute of Information Technology

Plot No. 30, Sector H-8/1, Islamabad 44000, PAKistan

E-mail address: nazirahmad.mir@gmail.com

ARIF RAFIQ

Department of Mathematics

COMSATS Institute of Information TeChNOLOGY

M. A. Jinnah Building Defense Road

LAHORE 54700, PAKISTAN

E-mail address: arafiq@comsats.edu.pk 